\title{
Dynamic Analysis of Sand-Clay Layered Ground Considering Viscous Effect of Clay
}

\author{
Kim, Yong-Seong*
}

\begin{abstract}
A cyclic viscoelastic-viscoplastic constitutive model for clay is incorporated into an effective stress based seismic response analysis to describe viscous effect of clay layer to sand layer during earthquake. The seismic response against main shock of 1995 Hyogoken Nambu Earthquake is analyzed in the present study. Acceleration responses in both clay layer and just upper liquefiable sand layer are damped due to viscous effect of clay. A cyclic viscoelastic-viscoplastic constitutive model for clay was implemented into a FEM code, and Newmark- $\beta$ method was employed for the time discretization in the finite element formulation. Seismic responses were simulated by numerical method with recorded data at Port Island, Kobe, Japan. As results of this study, it was found that a cyclic viscoelastic-viscoplastic constitutive model can give good description of dynamic behavior characteristics including viscoelastic effect.
\end{abstract}

Keywords : Clay, Dynamic analysis, Viscous effect, Constitutive model, Earthquake

\section{Introduction}

Since Niigata Earthquake in 1964, a large number of constitutive models for sand have been studied to describe the cyclic behavior and liquefaction of sands. Oka et al. (1999) also proposed a cyclic elasto-plastic model for sands based on nonlinear kinematic hardening rule. Considering that most of grounds in the Japanese

* Disaster Management Team, National Emergency Management Agency Seoul 110-760 Korea

* Corresponding author. Tel.: +82-2-2100-5412

Fax: +82-2-2100-5419

E-mail address: nakisuna(nema.go.kr water front are layered, effect of non-liquefiable clay layer to liquefiable sand layer is rather important to estimate the earthquake-resistant performance of the whole sand-clay layered ground.

Oka (1992) developed a cyclic elasto-viscoplastic constitutive model for clay based on non-linear kinematic hardening rule. Although this model is suited to describe a cyclic behavior of clay for the range of middle to high level strain, viscous effect of clay for the range of low level strain could not be expressed by the elastoviscoplastic model.

After the 1995 Hyogoken-Nambu earthquake, 
liquefaction phenomena were observed in the wide area, in particular, in reclaimed land such as Port Island, Rokko Island, etc. One of the issues of liquefaction analysis of sand-clay layered ground is the effect of clay layers on liquefaction of sandy layers. As for the dynamic analysis of ground during earthquake, it is necessary to account for the strain dependent characteristics of shear modulus and damping in the small strain level. Hence, in order to perform dynamic analysis accurately it is a better way to consider viscoelastic as well as viscoplastic characteristics of clay.

From this reason a viscoelastic-viscoplastic model for clay was developed, in which viscoelastic component is incorporated to the cyclic elasto-viscoplastic model, and we have shown that the viscoelastic-viscoplastic model could simulate the cyclic triaxial test of natural clay and strain level dependency of both shear modulus and hysteretic damping (Oka et al., 2004).

In the present study, the cyclic viscoelasticviscoplastic model for clay is installed to the effective stress based liquefaction analysis code, LIQCA-2D (Oka et al, 2004). Through the seismic response analysis for main shock in Port Island, effect of clay layer laying beneath liquefiable sand layer to dynamic properties of the whole sand-clay layered ground is discussed, in particular during relatively large earthquake.

\section{Viscoelastic-Viscoplastic Constitutive Model for Clay}

\section{Viscoelastic-Viscoplastic Constitutive Model}

model proposed in the present study is formulated by incorporating a three-parameter viscoelastic component into an elasto-viscoplastic constitutive model based on non-linear kinematic hardening rule, which was proposed by Oka et al. (1992). The three-parameter viscoelastic model, which combines a Voigt viscoelastic model and a linear elastic spring in series, has been used to describe a time dependent behavior of clay for the range of low level strain by several researchers (i.e. Hori, 1974; Di Benedetto and Tatsuoka, 1997; Oka et al., 2004).

In the proposed model, the viscoelastic properties are considered only in the deviatoric strain rate component, because it is difficult to distinguish between the compressive viscoelastic behavior of soil skeleton and pseudo viscoelastic behavior due to the interaction of water and soil skeleton. The viscoelastic deviatoric strain rate tensor, $e_{i j}^{i e}$, consist of an elastic deviatoric strain rate tensor, $e_{i j}^{e}$, and a Voigt type viscoelastic deviatoric strain rate tensor, $e_{i j}^{i e v}$, as

$$
\dot{e}_{i j}^{v e}=\dot{e}_{i j}^{e}+\dot{e}_{i j}^{v e v}=\frac{1}{2 G_{1}} S_{i j}+\frac{1}{\mu}\left(S_{i j}-2 G_{2} \cdot e_{i j}^{e v v}\right)
$$

where $s_{i j}$ and $s_{i j}$ are deviator stress tensor and deviator stress rate tensor, respectively. $G_{2}$ is an elastic shear modulus in the Voigt element and $G_{1}$ is the shear modulus of the other elastic spring, and $\mu$ is a viscous coefficient in the Voigt element. By adding an elastic volumetric strain rate and a vicoplastic strain rate tensor to $e_{i j}^{i e}$ in Eq. (1), the total strain rate tensor is given by

A cyclic viscoelastic-viscoplastic constitutive 


$$
\begin{aligned}
\dot{\varepsilon}_{i j} & =\dot{e}_{i j}^{i p}+\frac{1}{3} \ddot{\varepsilon}_{v}^{e} \delta_{i j}+\dot{\varepsilon}_{i j}^{v p} \\
& =\dot{e}_{i j}^{v p}+\frac{x}{3(1+e)} \frac{\ddot{\sigma_{m}}}{\sigma_{m}^{\prime}} \delta_{i j}+\dot{\varepsilon}_{i j}^{v p}
\end{aligned}
$$

The viscoplastic strain rate tensor is $\mathrm{ex}^{-}$ pressed as follows (Oka, 1992; Oka et al., 2004):

$$
\begin{aligned}
\dot{\varepsilon} u \dot{u p}= & C_{01}\left\langle\Phi_{1}\left(f_{y}\right)\right\rangle \frac{\eta_{i j}^{*}-\chi_{i j}^{*}}{\eta_{x}^{*}} \\
& +C_{02}\left\langle\Phi_{1}\left(f_{y}\right)\right\rangle\left(\bar{M}^{*}-\frac{\eta_{m n}^{*}\left(\eta_{m n}^{*}-\chi_{m n}^{*}\right)}{\eta_{x}^{*}}\right) \frac{\delta_{i j}^{*}}{3} \\
\overline{\eta_{x}^{*}}= & \left\{\left(\eta_{m n}^{*}-\chi_{m n}^{*}\right)\left(\eta_{m n}^{*}-\chi_{m n}^{*}\right)\right\}^{(1 / 2)}, \quad \eta_{i j}^{*}=\frac{S_{i j}}{\sigma_{m}}
\end{aligned}
$$

where, $C_{01}$ and $C_{02}$ are viscoplastic parameters, and Macaulay's bracket $\Longleftrightarrow$ expresses that $\langle x\rangle=x$, if $x\rangle 0,\langle x\rangle=0$, if $x \leq 0$. The static yield function, $f_{y}$ and the viscoplastic parameters $m$, $\Phi_{1}$ are assumed as follows (A dachi and Oka, 1982):

$$
\Phi_{1}\left(f_{y}\right)=\exp \left\{m f_{y}\right\}, f_{y}=\overline{\eta_{x}^{*}}=0
$$

Referring to Chaboche and Rousselier (1980), the non-linear kinematical hardening parameter, is defined by using the following evolutional equation:

$$
\begin{aligned}
& d \chi_{i j}^{*}=B_{1}^{*}\left(A_{1}^{*} d e_{i j}^{\nu p}-\chi_{i j}^{*} d \gamma^{v p}\right) . \\
& d \gamma^{v p}=\sqrt{d e_{i j}^{v p} d e_{i j}^{v p}} \ldots \ldots \ldots \ldots \ldots \ldots \ldots \ldots \ldots \ldots \ldots \ldots
\end{aligned}
$$

where $A_{1}$ is related to the stress ratio at failure, namely $A_{1}=M_{f}^{*}$ and $B_{1}$ is related to the viscoplastic shear modulus $G^{v p}$, namely $B_{1}=G^{v p} / M_{f}^{*}$ is varied corresponding to the viscoplastic shear strain $\gamma^{v p}\left(=\int d \gamma^{v p}\right)$ from initial value $B_{0}$ to the lower limit value $B_{s}$ as follows:

$$
B_{1}^{*}=B_{s}+\left(B_{0}-B_{s}\right) \exp \left(-B_{t} \gamma^{v p^{*}}\right)
$$

where $B_{t}$ is the decreasing rate of $B_{1}$.

\section{Fluid-Solid Mixture Theory}

Based on the definition of Terzaghi's effective stress, the total stress tensor is expressed as

$$
\sigma_{i j}^{T}=\sigma_{i j}+p \delta_{i j}
$$

where $\sigma_{i j}^{T}$ is the total stress tensor, $\dot{\sigma_{i j}}$ is the Terzaghi's effective stress tensor, $\mathrm{p}$ is the pore water pressure and $\delta$ is Kronecker's delta. The partial stresses are defined as

$$
\sigma_{i j}^{T}=\sigma_{i j}^{S}+\sigma_{i j}^{F} \cdots
$$

where $\sigma_{i j}^{S}$ is the partial stress of the solid phase and $\sigma_{i j}^{F}$ is the partial stress of the fluid phase. The partial stresses for two-phase mixture are referred to as

$$
\sigma_{i j}^{S}=\sigma_{i j}+(1-n) p \delta_{i j,} \quad \sigma_{i j}^{F}=n p \delta_{i j}
$$

where $\mathrm{n}$ is porosity and tensile stress is defined as positive.

The soil-water coupled dynamic governing equations are based on a $u-p$ formulation whose variables are the solid phase displacement and pore water pressure (Kim et. al., 2004). Finite element method (FEM) was used in the spatial discretization of the equilibrium equations, finite difference method (FDM) was used in the discretization of continuity equations and Newmark$\beta$ method was used for time integration. 


\section{Dynamic Analysis at Port Island}

Fig. 1 illustrates a soil profile at Port Island, where the heavy damage of civil structures occurred due to the 1995 Hyogoken Nambu Earthquake. This study applied the viscoelasticviscoplastic constitutive model of clay layer into two-dimensional analysis code (plane-strain condition) - LIQCA (Coupled Analysis of LIQuefaction) - developed by Oka et al. (1994), and applied Tangent Modulus method for the secure convergence of numerical analysis in doing so, it attempted to conduct an earthquake response analysis of multiple soil layers as a newly expanded and established LIQCA-2D(VE-VP) and to look into the role of seismic waves' effect on the behavior of clay layer. For the sand layers, a cyclic elasto-plastic model proposed by Oka et al. (1999) is used.

Figs. 2 and 3 show the obtained record of earthquake acceleration (GL.0.0m), which is NS component of horizontal direction and the input

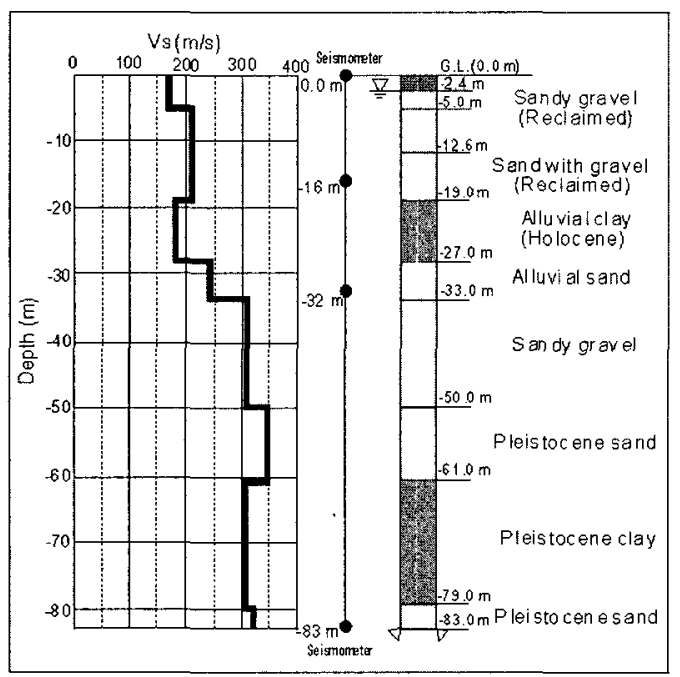

Fig. 1 Soil profile at Port Island in Japan

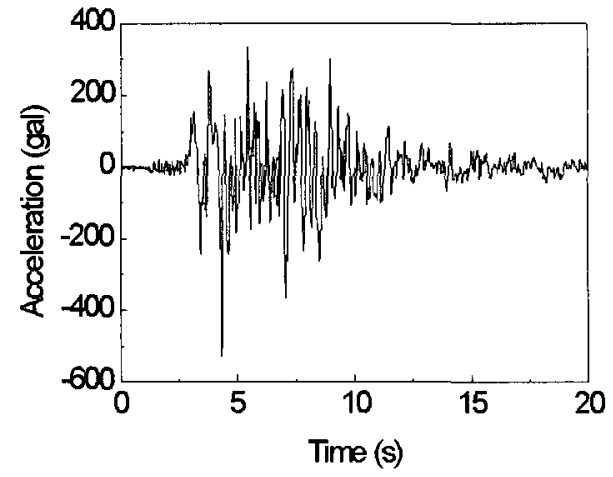

Fig. 2 Observed record at GL. $-83 \mathrm{~m}$

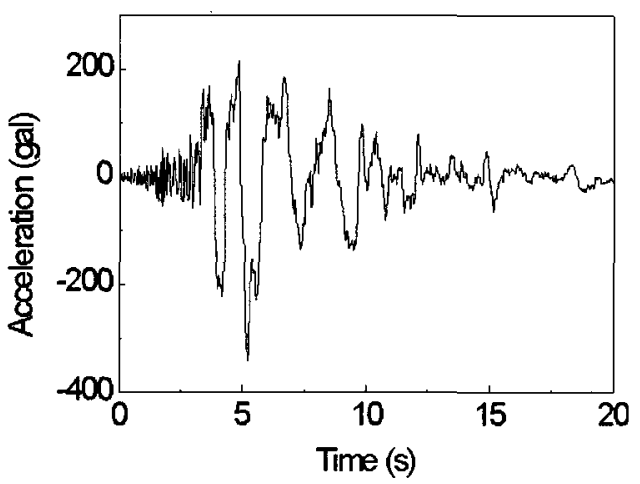

Fig. 3 Observed record at GL. $0.0 \mathrm{~m}$

motion (GL. $-83 \mathrm{~m}$ ). From the specification for the instrumented site at Port Island, Kobe, accelerometers were placed at four different depths. In the present analysis, all the predictions and comparisons were made at the three different depths, GL.0.0 m, $-16 \mathrm{~m}$ and $-32 \mathrm{~m}$, in NS direction.

In the present study, the acceleration at G.L. $-83 \mathrm{~m}$ was used as the input earthquake motion, and the seismic responses at various depths have been computed and compared to the records. We also compare between the analytical results by using the viscoelastic-viscoplastic model and the elasto-viscoplastic model for clay in order to 
estimate the viscoelastic effect of the clay layer for the range of low level strain. The acceleration measured during the principle Hyogoken Nanbu Earthquake amounted to 526.7 (gal) in Port Island GL. $-83 \mathrm{~m}$ and it was considered as a large-scale earthquake and it accompanied a liquefaction phenomenon and caused countless loss of life and property.

This study analyzed and compared the actually recorded seismic waves to the results of earthquake response analysis of two models such as elasto-viscoplastic model and viscoelastic-viscoplastic model divided in the earthquake response behavior in multiple soil layers including clay layer. The material constants used in this study referred to those of the existing researches (Oka et al., 2001) obtained from the numerical simulation of interior and exterior experiment and these could be summarized as in Table 1.

Loads in dynamic analysis can be considered as sine wave, seismic wave and the surface force on temporary element area (step wave type, sine wave type and spike wave type), but the actually measured seismic wave (GL.-83m, Port Island in Kobe) was used in the lowest-end fixed node of the soil model in this study. Here the soil model would become one-dimensional and the boundary section would meet at right angles with the incident wave; accordingly, the influence of a reflected wave would become a direct result of an earthquake response.

Meanwhile, the displacement of soil particles and transfer of pore water would be considered through the designation of boundary conditions. In general, it would be possible to take a single-

Table 1 Soil parameters used in the analysis at Port Island

\begin{tabular}{|c|c|c|c|c|c|c|c|c|c|c|}
\hline & $0-2.4$ & $2.4-5$ & $5-12.6$ & $12.6-19$ & $19-27$ & $27-33$ & $33-50$ & $50-61$ & $61-79$ & $79-83$ \\
\hline Parameters & Sand & Sand & Sand & Sand & Clay & Sand & Sand & Sand & Clay & Sand \\
\hline Compressional wave $\mathrm{Vp}(\mathrm{m} / \mathrm{s})$ & 260 & 330 & 780 & 1480 & 1180 & 1330 & 1530 & 1610 & 1610 & 2000 \\
\hline Shear wave $\mathrm{Vs}_{\mathrm{s}}(\mathrm{m} / \mathrm{s})$ & 170 & 170 & 210 & 210 & 180 & 245 & 305 & 350 & 303 & 320 \\
\hline Viscous parameters $\mu(\mathrm{kPa} \cdot \mathrm{s})$ & 0 & 0 & 0 & 0 & $5.0 \mathrm{E}+03$ & 0 & 0 & 0 & $5.0 \mathrm{E}+03$ & 320 \\
\hline Viscoplastic parameter $m^{\prime}$ & - & - & - & - & $2.0 \mathrm{E}-07$ & - & - & - & $1.0 \mathrm{E}-09$ & - \\
\hline Viscoplastic parameter $\mathrm{C}_{01}(1 / \mathrm{s})$ & - & - & - & - & $2.0 \mathrm{E}-09$ & - & - & - & $1.0 \mathrm{E}-11$ & - \\
\hline Viscoplastic parameter $\mathrm{C}_{02}(1 / \mathrm{s})$ & - & - & - & - & 20 & - & - & - & 20 & - \\
\hline Viscoplastic parameter $B_{0}$ & 100 & 100 & 70 & 500 & 50 & 1000 & 2000 & 3000 & 100 & 5000 \\
\hline Viscoplastic parameter $B_{s}$ & 1 & 1 & 1 & 1 & 1 & 1 & 1 & 1 & 1 & 1 \\
\hline Viscoplastic parameter $B_{t}$ & 1 & 1 & 1 & 1 & 1 & 1 & 1 & 1 & 1 & 1 \\
\hline Stress ratio at maximum compression $\mathrm{M}_{\mathrm{m}}{ }^{*}$ & 0.71 & 0.71 & 0.75 & 0.75 & 0.74 & 0.91 & 1.16 & 1.16 & 0.99 & 1.16 \\
\hline Stress ratio at failure state $\mathrm{M}_{\mathrm{f}}{ }^{*}$ & 1.01 & 1.01 & 1.05 & 1.05 & 1.24 & 1.21 & 1.41 & 1.57 & 1.24 & 1.57 \\
\hline Compression index $\lambda$ & 0.03 & 0.3 & 0.03 & 0.3 & 0.39 & 0.02 & 0.02 & 0.02 & 0.34 & 0.03 \\
\hline Swelling index $\mathrm{k}$ & 0.00026 & 0.00027 & 0.00054 & 0.00072 & 0.05 & 0.00133 & 0.0011 & 0.00114 & 0.00261 & 0.00203 \\
\hline Poisson's ratio $u$ & 0.25 & 0.25 & 0.25 & 0.25 & 0.488 & 0.25 & 0.25 & 0.25 & 0.3 & 0.25 \\
\hline Initial void ratio en & 0.6 & 0.6 & 0.6 & 0.6 & 1.5 & 0.6 & 0.5 & 0.5 & 1.2 & 0.5 \\
\hline
\end{tabular}




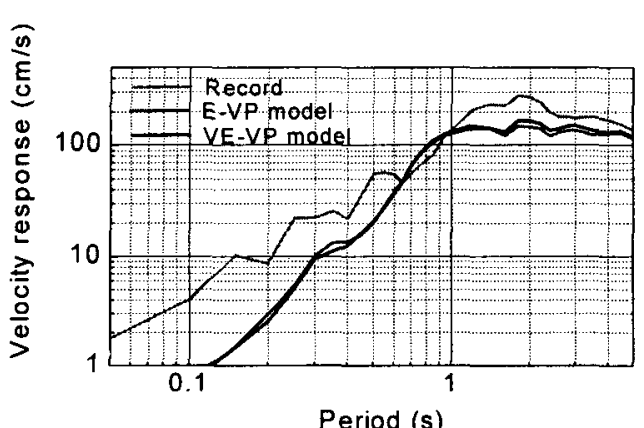

(a) G.L.0.0m Reclaimed Land

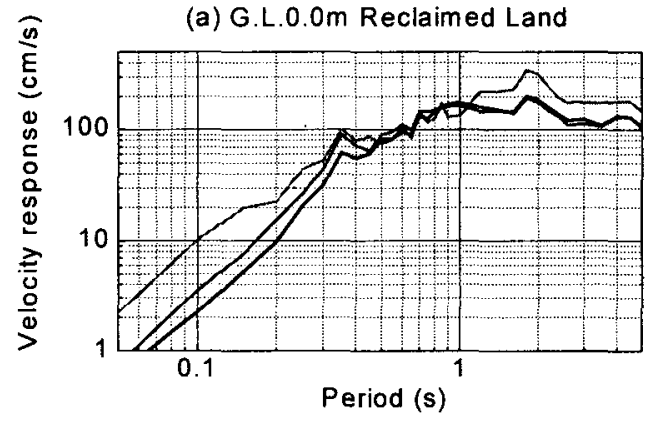

(b) G.L.-16.0m Reclaimed Land

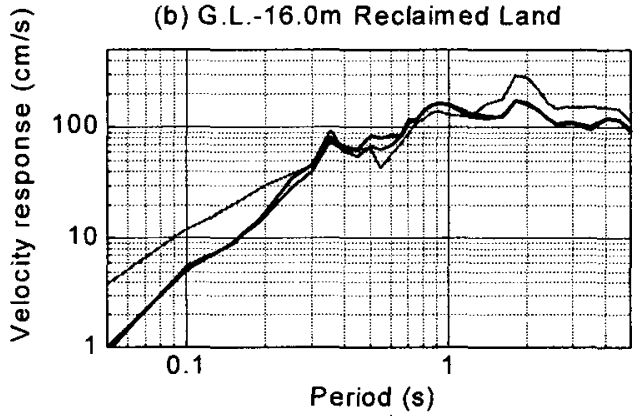

(c) G.L.-32.0m Sand

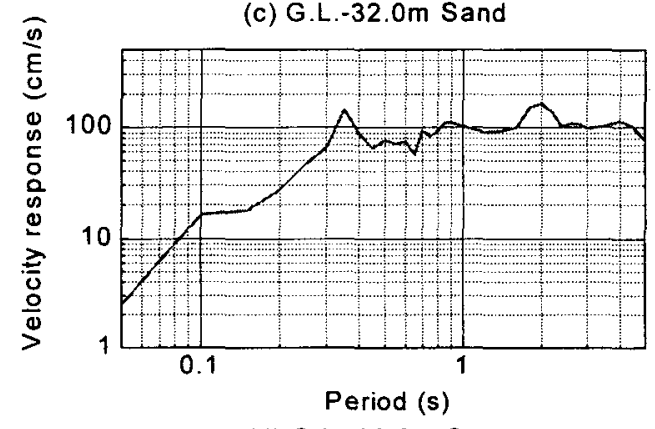

(d) G.L. $-83.0 \mathrm{~m}$ Sand

Fig. 4 Velocity response spectrum

point constraint and simple multi point constraint for the displacement of soil particles, and it would be possible to be given the constant displacement

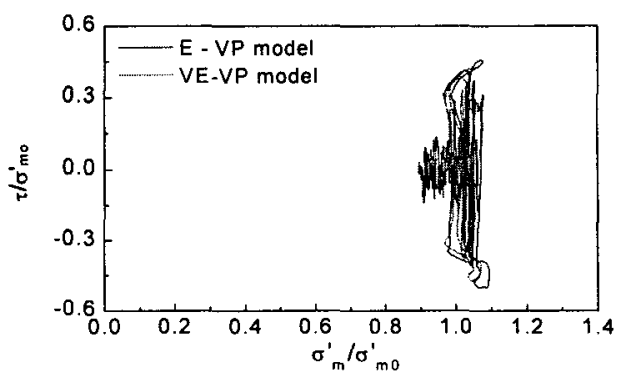

(a) Effective stress ratio vs. shear stress ratio

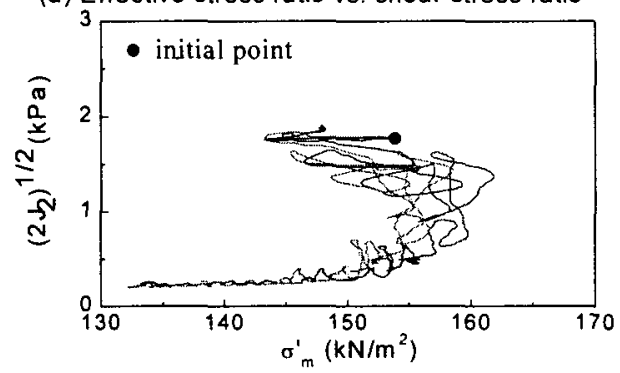

(b) Effective stress path

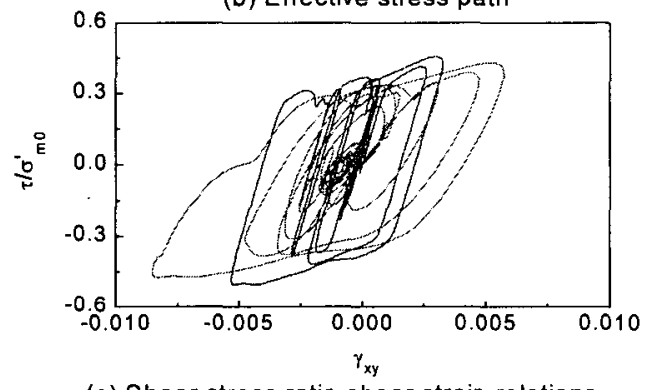

(c) Shear stress ratio-shear strain relations

Fig. 5 Stress path and stress-strain relations in clay at GL. $-24.25 \mathrm{~m}$

boundary conditions that would identify temporary 2-node displacement in multi point constraint. For the transfer of interstitial water, the side of the temporary element would be as drained or undrained boundary.

Besides, viscous boundary could be set up on the sides or lower area to reproduce the boundlessness of analysis scope. The analysis code would be able to express the energy dissipation by Rayleigh damping, hysteresis damping and viscous boundary; however, this study has not used the viscous boundary as the constant 
displacement boundary conditions of the onedimensional model. Meanwhile, one of the advantages of this analysis would lie in the fact that the damping effects subsequent to ground materials could be applied to the characteristics of their hysteretic damping.

Fig. 4 shows the velocity response analysis with the condition of $2 \%$ damping ratio at Port Island, and it is seen that Figs. 4(b) and (c) give quite good overall descriptions of the velocity response by the proposed model with the observed records, even though Figs. 4(b) and (c) shows slightly different velocity response at the low period region. On the other hand, Fig. 4 (a) shows a quite low velocity in the region of under 1 second period, but gives a good description of the velocity response at the region of over 1 second period.

Fig. 5 shows stress path and stress-strain relations in the clay layer at GL. $-24.25 \mathrm{~m}$ calculated by the elasto-viscoplastic constitutive model and viscoelastic-viscoplastic constitutive model. The difference between two models comes from the effect of clay behavior in the small strain level. The area of hysteretic loop of elastic-viscoelastic model case is smaller than that of viscoelastic-viscoplastic model case. The elastic-viscoplastic model behaves rather elastically in small strain level while the viscoelastic-viscoplastic model exhibits viscoelastic behavior in small strain level.

Therefore, it has been considered that it would be more appropriate to apply the cyclic viscoelastic-viscoplastic model that could analyze the dampening characteristics of clay layer during large earthquake occurring in the multiple soil layers.

\section{Conclusion}

This study has performed an earthquake response analysis on the multiple soil layers using a two-dimensional analysis code (plane-strain conditions) - LIQCA-2D(VE-VP) - that applied the cyclic viscoelastic-viscoplastic constitutive model of clay layer and has investigated the role of seismic wave in the behavior of clay layer. Followings are the main conclusions drawn from this study.

1) From the velocity response analysis with the condition of $2 \%$ damping ratio at Port Island, the cyclic viscoelastic-viscoplastic constitutive model gives quite good overall descriptions of the velocity response at $\mathrm{GL} .-16 \mathrm{~m}$ and $-32 \mathrm{~m}$ with slightly different velocity response at the low period region, and it shows a quite low velocity in the region of under 1 second period at ground level but gives a good description of the velocity response at the region of over 1 second period.

2) From the simulation of stress path and stress-strain relations in the clay layer, the area of hysteretic loop of elastic-viscoelastic model case is smaller than that of viscoelastic-viscoplastic model case.

3) It would be more appropriate to apply the cyclic viscoelastic-viscoplastic model that could analyze the dampening characteristics of clay layer during large earthquake occurring in the multiple soil layers.

\section{References}

1. Di Benedetto, H. and F. Tatsuoka. 1997. Small Strain Behavior of Geomaterials: Modelling of Strain Rate Effects. Soils and Foundations 37(2): 127-138 
2. Hori, M. 1974. Fundamental Studies on Wave Propagation Characteristics Through Soils. Ph.

D. Dissertation. Dept. of Civil Engrg. Kyoto Univ. Kyoto. Japan.

3. Kim, Y.-S. 2006. Dynamic Behavior Characteristics of Clay in Wide Strain Range Based on Viscoelastic-Viscoplastic Constitutive Model. International Journal of Offshore and Polar Engineering 16(2): 153-160.

4. Kim, Y.-S. and D.-W. Lee. 2004. Evaluation Technique of Seismic Performance on Agricultural Infrastructure. Journal of the Korean Society of Agricultural Engineers 46(4): 75-84. (in Korean)

5. Oka, F. 1992. A Cyclic Elasto-Viscoplastic Constitutive Model for Clay Based on the Non-Linear Hardening Rule. Proc. 4th Int. Symp. on Numerical Models in Geomechanics 1: 105-114.

6. Oka, F., Kodaka, T. and Y.-S. Kim. 2001. A Cyclic Viscoelastic-Viscoplastic Model for
Clay and Its Application to Liquefaction Analysis of Ground. Proc. 10th Int. Symp. on Computer Methods and Advances in Geomechanics 2: 1025-1031.

7. Oka, F., Kodaka, T. and Y.-S. Kim. 2004. A Cyclic Viscoelastic-Viscoplastic Model for Clay and Liquefaction Analysis of Multilayered Ground. International Journal for $\mathrm{Nu}$ merical and Analytical Methods in Geomechanics 28(2): 131-179.

8. Oka, F., Yashima, A., Shibata, T., Kato, M. and Uzuoka, R. 1994. FEM-FDM Coupled Liquefaction Analysis of a Porous Soil Using an Elasto-Plastic Model. Applied Scientific Research 52: 209-245.

9. Oka, F., Yashima, A., Tateishi, A., Taguchi, Y. and Yamashita, S. 1999. A Cyclic ElastoPlastic Constitutive Model for Sand Considering a Plastic-Strain Dependence of the Shear Modulus. Geotechnique 49(5): 661-680. 